\title{
Self-Consistent Optics with Strong Space Charge ${ }^{1}$
}

\author{
Y. Alexahin, E. Stern \\ Fermi National Accelerator Laboratory, PO Box 500, Batavia, IL 60510, USA \\ A. Prieto Tirado \\ University of Cantabria, Avenida de los Castros, s/n, 39005 Santander, Cantabria, Spain
}

\begin{abstract}
A method for finding self-consistent beam optics in the presence of strong space charge defocusing effect is developed which is based on the eigenvectors of the transfer matrix. It allowed us to obtain solutions for the $\Sigma$-matrix in the case of the space charge tune depression as high as $25 \%$. These solutions were used to build a matched initial distribution of particles for tracking simulations with the Synergia code [1]. The results of simulations are presented and discussed.
\end{abstract}

PACS numbers: 29.27.Bd, 29.27.Fh

\section{Introduction}

The space charge (SC) of the beam exerts a defocusing effect on the beam particles changing the beam sizes and the SC strength. Our goal is to find a self-consistent solution for the r.m.s. beam sizes $\sigma_{x}, \sigma_{y}$ around the lattice that render the exact strength of the SC (de)focusing needed to obtain these beam sizes. We are interested in stationary solution which can be particularly important for tracking simulations to avoid large initial mismatch.

\section{Basic Theory}

We will use the arc length $s$ as the independent variable and follow the MAD convention [2] for the dynamical variables normalizing the transverse canonical momenta by the reference momentum $p_{0}=m c \beta_{0} \gamma_{0}$ and using for the longitudinal plane canonical variables $-c t$, and

$$
p_{t}=\frac{\gamma-\gamma_{0}}{\beta_{0} \gamma_{0}} .
$$

The phase space vectors will be marked with an underscore

\footnotetext{
${ }^{1}$ This manuscript has been authored by Fermi Research Alliance, LLC under Contract No. DE-AC02-07CH11359 with the U.S. Department of Energy, Office of Science, Office of High Energy Physics.
} 


$$
\underline{z}=\left(x, p_{x}, y, p_{y},-c t, p_{t}\right)
$$

Propagation of a phase space vector from point $s_{1}$ to point $s_{2}$ along the lattice of linear (or linearized) elements can be described with transfer matrix

$$
\underline{z}\left(s_{2}\right)=\mathrm{T} \underline{z}\left(s_{1}\right)
$$

\section{Eigensystem of the transfer matrix.}

The 1-turn transfer matrix of a linearly stable lattice has three complex-conjugate pairs of eigenvalues and eigenvectors

$$
\mathrm{T} \underline{v}_{k}=\mu_{k} \underline{v}_{k}, \quad \mu_{2 m}=\mu_{2 m-1}^{*}, \quad \underline{v}_{2 m}=\underline{v}_{2 m-1}^{*}
$$

where $m=1,2,3$ is the mode number, asterisk denotes complex conjugation, underscore means 6D vector. The eigenvalues are related to the normal mode tunes $Q_{m}$ as $\mu_{2 m-1}=\exp \left(2 \pi i Q_{m}\right)$.

We choose normalization as in MAD

$$
\left(\underline{v}_{2 m-1}^{*}, \mathbf{S} \underline{v}_{2 m-1}\right)=2 i
$$

where

$$
\mathrm{S}=\left(\begin{array}{cccccc}
0 & 1 & 0 & 0 & 0 & 0 \\
-1 & 0 & 0 & 0 & 0 & 0 \\
0 & 0 & 0 & 1 & 0 & 0 \\
0 & 0 & -1 & 0 & 0 & 0 \\
0 & 0 & 0 & 0 & 0 & 1 \\
0 & 0 & 0 & 0 & -1 & 0
\end{array}\right)
$$

Condition (5) can be rewritten for real and imaginary parts of the eigenvectors as

$$
\begin{aligned}
& \left(\underline{v}_{i}^{\prime}, \mathrm{S} \underline{v}_{j}^{\prime}\right)=\left(\underline{v}_{i}^{\prime \prime}, \mathrm{S} \underline{v}_{j}^{\prime \prime}\right)=0, \\
& \left(\underline{v}_{2 m-1}^{\prime}, \mathrm{S} \underline{v}_{2 n-1}^{\prime \prime}\right)=\delta_{m n}, \\
& \underline{v}_{i}^{\prime} \equiv \operatorname{Re} \underline{v}_{i}, \quad \underline{v}_{i}^{\prime \prime} \equiv \operatorname{Im} \underline{v}_{i}
\end{aligned}
$$

Any phase space vector can be expanded in eigenvectors

$$
\underline{z}=\operatorname{Re} \sum_{m=1}^{3} \underline{v}_{2 m-1} \sqrt{2 J_{m}} e^{i \psi_{m}}
$$

with $J_{m}, \psi_{m}$ being the action-angle variables. In a linear system the action variables $J_{m}$ are integrals of motion and so are their average values over an ensemble of particles, called the geometrical r.m.s. emittances:

$$
\varepsilon_{m}=\left\langle J_{m}\right\rangle \equiv \frac{1}{N} \sum_{i=1}^{N} J_{m}^{(i)}
$$




\section{$\underline{\sum \text {-matrix }}$}

The main instrument that we will use is the covariance matrix ( $\Sigma$-matrix) defined for a sample of $N$ particles with phase space coordinates $\underline{z}=\left(x, p_{x}, y, p_{y},-c t, p_{t}\right)$ as

$$
\Sigma_{i j}=\frac{1}{N} \sum_{k=1}^{N} \zeta_{i}^{(k)} \zeta_{j}^{(k)}, \quad \underline{\zeta}^{(k)}=\underline{z}^{(k)}-\underline{a}, \underline{a}=\frac{1}{N} \sum_{k=1}^{N} \underline{z}^{(k)}
$$

It is clear that $\Sigma_{11}$ and $\Sigma_{33}$ are squares of the r.m.s. beam sizes $\sigma_{x}, \sigma_{y}$.

For a random particle distribution the angle variables are statistically independent and in the limit $N \rightarrow \infty$ we have

$$
\left\langle\cos \psi_{m} \cos \psi_{n}\right\rangle=\left\langle\sin \psi_{m} \sin \psi_{n}\right\rangle=\frac{1}{2} \delta_{m n}
$$

with $\delta_{m n}$ being the Kronecker delta.

Substituting ansatz (8) into definition (10) and using (11) we obtain in the limit $N \rightarrow \infty$

$$
\Sigma_{i k}=\sum_{m=1}^{3} \varepsilon_{m}\left(v_{2 m-1, i}^{\prime} v_{2 m-1, k}^{\prime}+v_{2 m-1, i}^{\prime \prime} v_{2 m-1, k}^{\prime \prime}\right),
$$

where $v_{m, k}$ means the $k$-th component of the $m$-th eigenvector.

Based on eq. (12) and orthonormality conditions (7) one can show that the matrix product $\Sigma \mathrm{S}$ has the same set of eigenvectors as the 1-turn transfer matrix but different eigenvalues

$$
\Sigma \mathrm{S}_{k}=\lambda_{k} \underline{v}_{k}, \quad \lambda_{2 m-1}=i \varepsilon_{m}, \quad \lambda_{2 m}=\lambda_{2 m-1}^{*}, \quad m=1,2,3
$$

This fact can be used for finding emittances (and optics functions) from tracking data.

Equation (12) can be considered as the extension of the classical definition of beam sizes

$$
\sigma_{x}=\left[\beta_{x} \varepsilon_{x}+\left(D_{x} \sigma_{p t}\right)^{2}\right]^{1 / 2}, \quad \sigma_{y}=\left[\beta_{y} \varepsilon_{y}\right]^{1 / 2},
$$

on the case of fully coupled 6D motion.

Propagation of the $\sum$-matrix

The transport of the $\Sigma$-matrix along a linear lattice is performed with the help of transfer matrices $\mathrm{T}$ as

$$
\Sigma^{(2)}=\mathrm{T} \cdot \Sigma^{(1)} \cdot \mathrm{T}^{\mathrm{t}},
$$

where superscript " $t$ " means transposition, $\mathrm{T}$ is the transfer matrix from point 1 to point 2 .

Generally the SC force is nonlinear, however, if we are interested in the $\Sigma$-matrix dynamics only we can invoke the fundamental result by F. Sacherer [3] for a coasting beam that for all transverse beam density profiles that depend on bilinear form 


$$
\rho=f\left(\frac{x^{2}}{\sigma_{x}^{2}}+\frac{y^{2}}{\sigma_{y}^{2}}\right)
$$

the effective quadrupole gradients are

$$
k_{1}^{(x)}=\frac{\kappa}{\sigma_{x}\left(\sigma_{x}+\sigma_{y}\right)}, \quad k_{1}^{(y)}=\frac{\kappa}{\sigma_{y}\left(\sigma_{x}+\sigma_{y}\right)},
$$

where

$$
\kappa=\frac{r_{p} N^{\prime}}{\beta_{0}^{2} \gamma_{0}^{3}}
$$

with $N^{\prime}$ being the linear density of particles, $r_{p}=1.5347 \cdot 10^{-18} \mathrm{~m}$. The SC strength $\kappa_{0}$ seen by particles with small deviations depends on the beam distribution function. For a $\mathrm{K}-\mathrm{V}$ distribution $\kappa_{0}$ is given by the same eq. (18) while for a Gaussian beam it is twice that value.

In a Gaussian bunched beam the linear density at the center is $N^{\prime}=N_{b} / \sqrt{2 \pi} \sigma_{z}, N_{b}$ being the number of particles in the bunch, but we also must perform averaging over the bunch length obtaining finally

$$
\kappa=\frac{1}{2^{3 / 2}} \kappa_{0}=\frac{r_{p} N_{b}}{2 \sqrt{\pi} \sigma_{z} \beta_{0}^{2} \gamma_{0}^{3}},
$$

with $\kappa_{0}$ being the SC strength seen by particles with small deviations from the center of a Gaussian (in all directions) bunch.

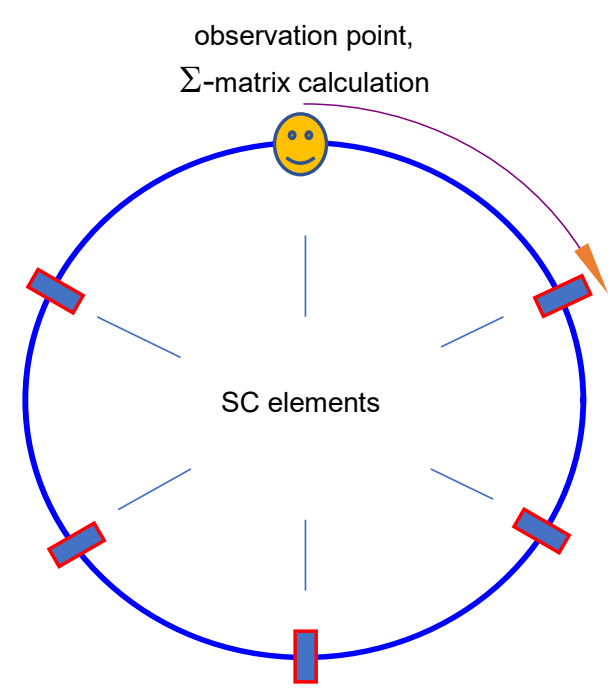

Figure 1. Schematic of SC simulations

\section{Space Charge Simulations}

The beam is represented by a number of thin elements producing SC kicks. PIC (Particle-In-Cell) codes like Synergia [1] compute the SC density form the tracking particle distribution whereas "express" codes like MADX-SC [4] rely on analytical formulas with periodically updated r.m.s. beam sizes.

Our purpose here is to find a stationary self-consistent solution for the $\Sigma$-matrix which would provide i) the initial approximation for the r.m.s. beam sizes $\sigma_{x}, \sigma_{y}$ at $\mathrm{SC}$ elements for MADX-SC simulations and ii) a matched $\Sigma$ matrix at the start of the lattice to generate the initial particle distribution for tracking with both Synergia and MADXSC. 
The total transfer matrix is a concatenation of transfer matrices $\mathrm{R}_{k}$ for segments between the SC elements and transfer matrices $\mathrm{B}_{k}$ over the $\mathrm{SC}$ elements:

$$
\mathrm{T}=\mathrm{R}_{n_{\mathrm{SC}}+1} \cdot \mathrm{B}_{n_{\mathrm{SC}}} \cdot \mathrm{R}_{n_{\mathrm{SC}}} \cdot \ldots \cdot \mathrm{B}_{1} \cdot \mathrm{R}_{1}
$$

where $n_{\mathrm{SC}}$ is the total number of SC elements.

We limit ourselves to the case of no transverse coupling so that there is no tilt of the beam ellipse. The possibility of such tilt can be added in the future. Also, we do not consider the effect of SC longitudinal field.

Transport of the $\Sigma$-matrix over a thin SC element is simplified by the fact that particle positions do not change during the kick so that the only non-zero elements in the corresponding transfer matrix B are

$$
B_{n n}=1, \quad n=1, \ldots, 6, \quad B_{21}=\frac{\kappa L_{k}}{\sigma_{x}\left(\sigma_{x}+\sigma_{y}\right)}, \quad B_{43}=\frac{\kappa L_{k}}{\sigma_{y}\left(\sigma_{x}+\sigma_{y}\right)}
$$

where $L_{k}$ is the length of the lattice ascribed to the $k$-th SC element. At each pass through the lattice the beam sizes $\sigma_{x}, \sigma_{y}$ change thus changing the overall transfer matrix (20).

Fixed point search

The search for a stationary self-consistent solution can be understood as search for a matrix $\Sigma$ that is invariant under transformation (15) with the transfer matrix (20). Without an artificial damping the $\Sigma$-matrix will exhibit sustained oscillations (which are in fact the beam envelope oscillations) without converging to a stationary value. The damping can be introduced e.g. in the following way:

$$
\Sigma^{(i+1)}=d \Sigma^{(i)}+(1-d) \mathrm{T}_{i} \cdot \Sigma^{(i)} \cdot \mathrm{T}_{i}^{\mathrm{t}}
$$

where $0<d<1$ is the damping factor and $i$ is the iteration number.

There is a number of complications with this approach, first of all the emittances are not controlled, so even in the case when the iterations do converge the found solution may correspond to different emittances from the desired ones so an external iteration loop over the emittances should be added.

Symplectic iterations

In principle, iteration can be done in a symplectic manner so that the emittances were preserved. Let $\mathrm{T}_{i}$ be the overall transfer matrix at $i$-th iteration. Using its eigenvectors we can build matrix

$$
\tilde{\mathrm{T}}(\theta)=-\frac{i}{2} \mathrm{~V} \Lambda(\theta) \mathrm{S} \mathrm{V}^{\mathrm{t}} \mathrm{S}
$$

where $\mathrm{V}$ is a matrix composed of the $\mathrm{T}$ eigenvectors as its columns, and

$$
\Lambda(\theta)=\operatorname{diag}\left(e^{i\left\{Q_{1}\right\} \theta}, e^{-i\left\{Q_{1}\right\} \theta}, e^{i\left\{Q_{2}\right\} \theta}, e^{-i\left\{Q_{2}\right\} \theta}, e^{i\left\{Q_{3}\right\} \theta}, e^{-i\left\{Q_{3}\right\} \theta}\right),
$$

is a diagonal matrix with $\left\{Q_{m}\right\}=Q_{m}-\left[Q_{m}\right]$ being distance to the nearest integer $\left[Q_{m}\right],-1 / 2<\left\{Q_{m}\right\} \leq 1 / 2$. 
It can be verified that matrix $\tilde{\mathrm{T}}$ is symplectic for all parameters $\theta$ and with $\theta$ changing from 0 to $2 \pi$ it is changing from the identity matrix to T. Now we can use $\tilde{\mathrm{T}}$ with sufficiently small $\theta$ for emittancepreserving iteration

$$
\Sigma^{(i+1)}=\tilde{\mathrm{T}}_{i}(\theta) \cdot \Sigma^{(i)} \cdot \tilde{\mathrm{T}}_{i}^{\mathrm{t}}(\theta) .
$$

This method has some drawbacks too. Since the tunes are not controlled, in the case of strong SC at some step the tunes may get into a linear resonance stopband breaking the process.

\section{$\underline{\text { Multiple solutions }}$}

The nonlinear dependence of the transfer matrix B on the $\Sigma$-matrix brings about the possibility of existence of multiple solutions [5] that are stationary and closed ${ }^{2}$. The problem is that in the vicinity of well-behaved solution the Banach contractive mapping theorem may not hold so that the iterations will diverge from it. We will give such an example later.

In such situation Newton's or secant method can be helpful.

Newton's method

The derivatives of the effective gradients (17) w.r.t. the $\Sigma$-matrix elements can be calculated analytically making it easy to implement Newton's algorithm.

Let us find some small variation $\xi$ of the current approximation $\Sigma_{0}$ that will make $\Sigma=\Sigma_{0}+\xi$ invariant under transformation (15), i.e.

$$
\mathrm{T} \cdot \Sigma \cdot \mathrm{T}^{\mathrm{t}}(=\Sigma)=\Sigma_{0}+\xi
$$

On the other hand, expanding the 1.h.s. of this equation we get

$$
\mathrm{T} \cdot \Sigma \cdot \mathrm{T}^{\mathrm{t}}=\mathrm{T} \cdot \Sigma_{0} \cdot \mathrm{T}^{\mathrm{t}}+\mathrm{T} \cdot \xi \cdot \mathrm{T}^{\mathrm{t}}+\Sigma_{0} \cdot\left(\nabla \mathrm{T} \cdot \xi \cdot \mathrm{T}^{-1}\right)^{\mathrm{t}}+\left(\nabla \mathrm{T} \cdot \xi \cdot \mathrm{T}^{-1}\right) \cdot \Sigma_{0} .
$$

Equating the r.h.s. of (26) and (27) we obtain a matrix equation for the necessary variation $\xi$

$$
\xi-\mathrm{T} \cdot \xi \cdot \mathrm{T}^{\mathrm{t}}-\Sigma_{0} \cdot\left(\nabla \mathrm{T} \cdot \xi \cdot \mathrm{T}^{-1}\right)^{\mathrm{t}}-\left(\nabla \mathrm{T} \cdot \xi \cdot \mathrm{T}^{-1}\right) \cdot \Sigma_{0}=\mathrm{T} \cdot \Sigma_{0} \cdot \mathrm{T}^{\mathrm{t}}-\Sigma_{0} .
$$

This method proves to be quite efficient for finding well-behaved solutions when the Banach fixed-point criterion is not fulfilled. Again, iteration of emittances may be necessary. It may be possible however to impose additional constraints on $\xi$ to render transformation $\Sigma_{0} \rightarrow \Sigma_{0}+\xi$ symplectic.

\section{"Toy Lattice" Simulations}

The effects of strong SC $\left(\left|\Delta Q_{\perp}\right| \sim 0.9\right)$ were studied in the case of 12-cell FODO lattice without bends and sextupoles. $20 \mathrm{SC}$ elements were installed in each $24 \mathrm{~m}$ long cell. The beam kinetic energy was $0.8 \mathrm{GeV}$, bare lattice tunes were $Q_{x}=3.72, Q_{y}=3.845, Q_{s}=0.077$ so that the SC tune depression was $\sim 25 \%$. The r.m.s. beam emittances were $\varepsilon_{\perp}=1 . \mathrm{e}-6 \mathrm{~m}, \varepsilon_{L}=0.00147 \mathrm{~m}$ $\left(\sigma_{s}=0.5 \mathrm{~m}\right)$. The number of particles per bunch was $N_{b}=2 . \mathrm{e} 11$, so that $\kappa_{0}=1.076 \mathrm{e}-7$.

\footnotetext{
${ }^{2}$ I.e. having periodicity at least 1 , not necessarily the lattice periodicity.
} 

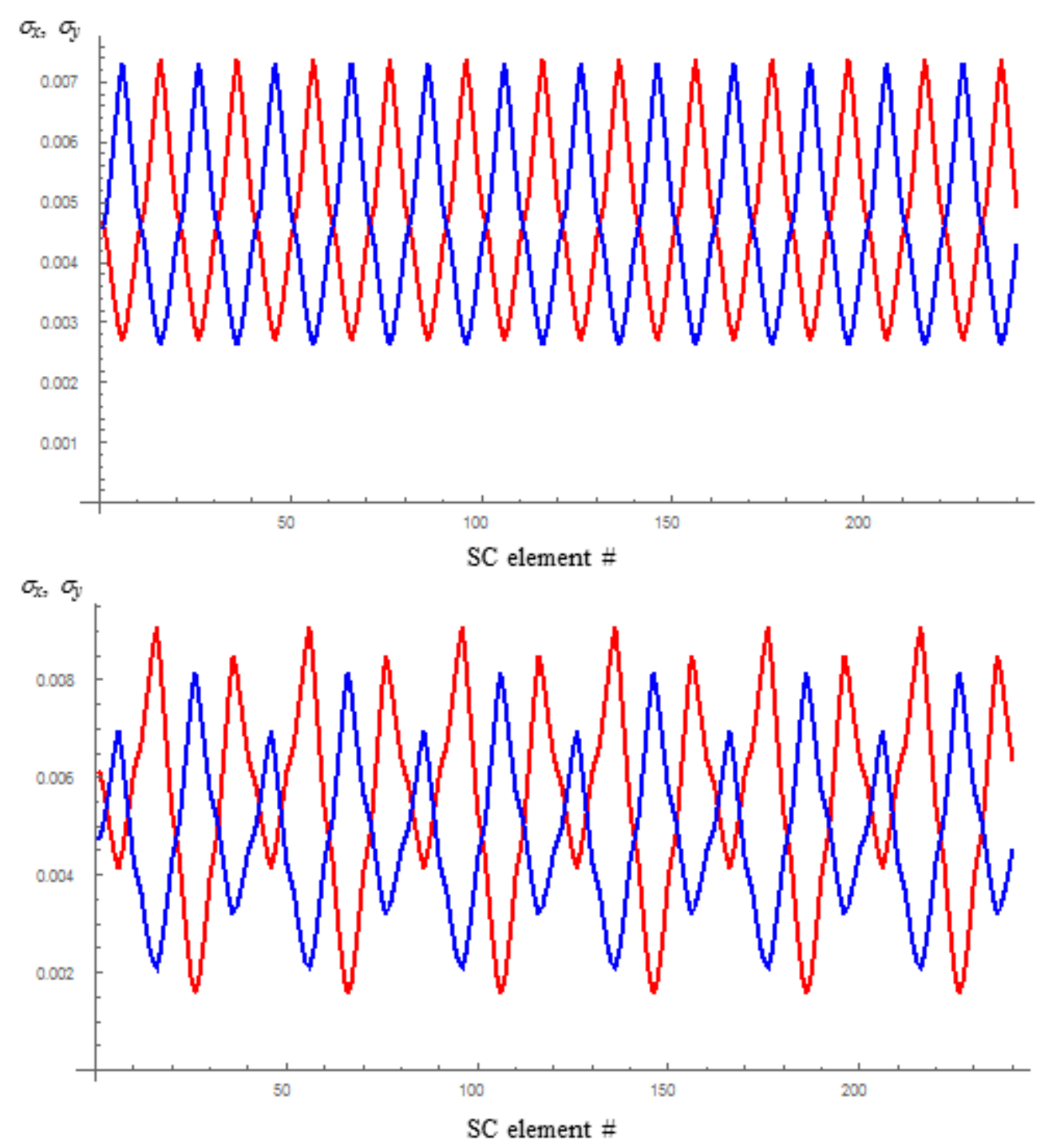

Figure 2. Self-consistent beam r.m.s. sizes (red - horizontal, blue - vertical) in the periodic lattice with ultimate SC strength.

\section{$\underline{\text { Self-consistent } \Sigma \text {-matrix }}$}

As a preliminary study we looked at the self-consistent $\Sigma$-matrix for various values of the SC strength parameter $\kappa$. Eq. (19) implies averaging of SC kicks over all three degrees of freedom. This may not provide the best matching, since it is possible that matching for small amplitude particles is more important than matching on average. So we tried larger $\kappa$ values, first $\kappa / \kappa_{0}=$ $1 / 2$ (absence of averaging over the bunch length), then $\kappa / \kappa_{0}=1$ (no averaging at all). The latter case will be referred to as the "ultimate" SC strength.

At small values of the SC strength parameter $\kappa$ both fixed point search and Newton's algorithm converge to the same well-behaved solutions. The problem arises at high SC strength in the presence of optics perturbation. 
In the perfectly periodic lattice it was possible to find a periodic solution with lattice periodicity (Fig. 2 top) by simply repeating the solution found for one cell. Starting from this solution (with a small error) the fixed point search for the whole lattice quickly departed from it converging to a completely different solution of periodicity 6 (Fig. 2 bottom). It corresponds to out-of-phase envelope oscillations which tuneshift was depressed enough to put the total envelope tunes on integer $(=3)$ thus ensuring $\Sigma$-matrix periodicity over the whole lattice length. It is quite possible that there can be more such solutions.

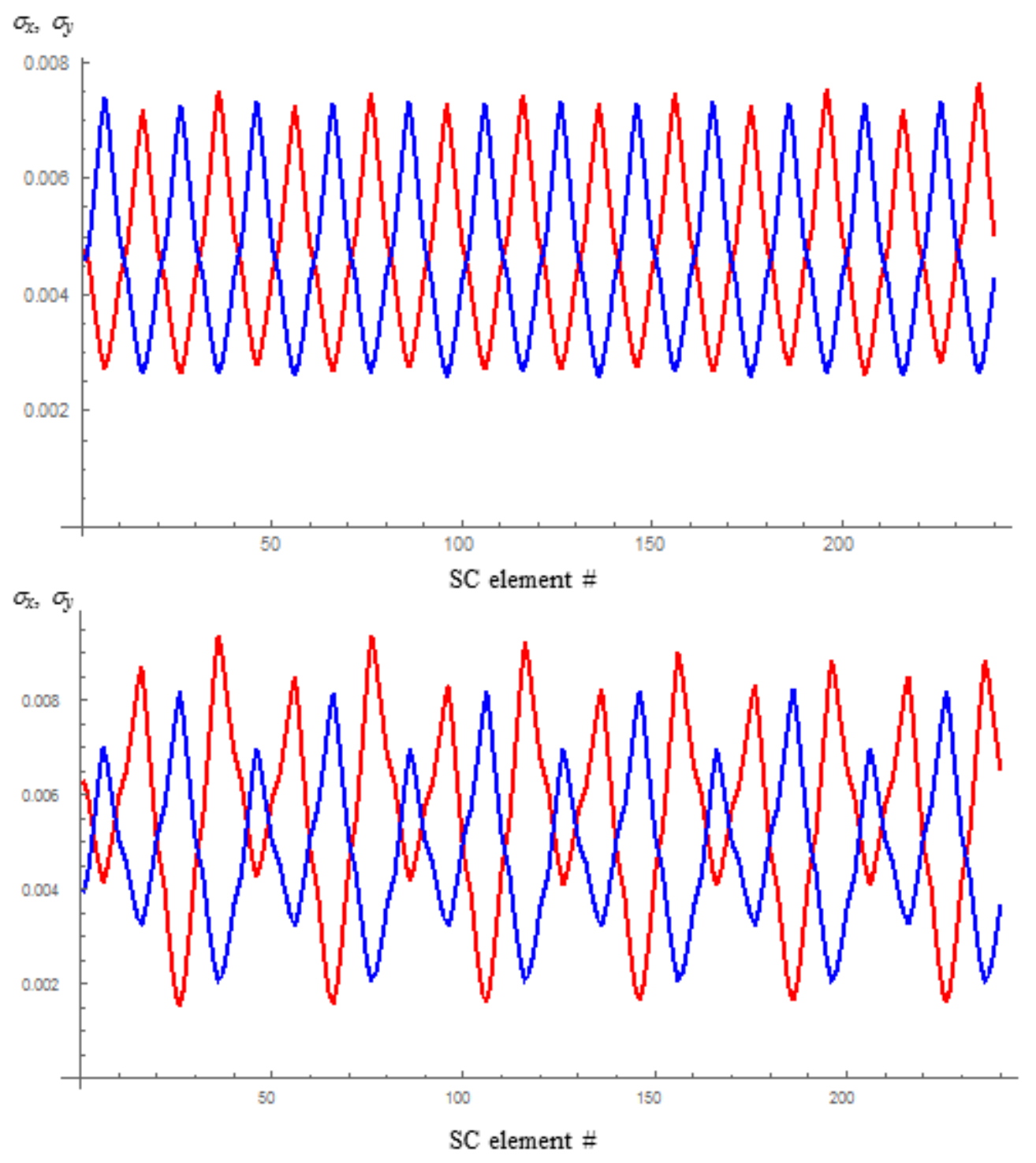

Figure 3. Self-consistent beam r.m.s. sizes (red - horizontal, blue - vertical) in the perturbed lattice with ultimate SC strength.

When a perturbation was introduced by increasing the strength of the first focusing quadrupole by $1 \%$ it was possible to find a well-behaved solution (almost periodic with lattice periodicity) in the case of low SC strength. But with the "ultimate" SC strength such solution apparently did not exist, the best found approximation to use for tracking with Synergia is shown in Fig. 3 (top). 
The emittance-preserving method (25) converged to a solution of periodicity 1 (Fig. 3 bottom) with amplitude of envelope oscillations only slightly higher than in the case of ideal lattice.

From this analysis we can draw the following conclusions:

1. There are three regions of the SC strength regarding the self-consistent beam sizes: at low strength there is a single stationary solution; at medium strength there still exists a well-behaved solution while other solutions appear corresponding to large-amplitude envelope oscillations; at high strength the well-behaved solution disappear leaving only the one(s) with large-amplitude envelope oscillations.

2. The envelope resonances manifest themselves as these large-amplitude envelope oscillations. They do not lead to unbound amplitude growth because of nonlinear detuning.

It should be stressed the last observation pertains only to the envelope oscillations. In a real beam with tune spread of individual particles there can be so-called particle-core resonance [6] leading to halo formation and increase in emittance.

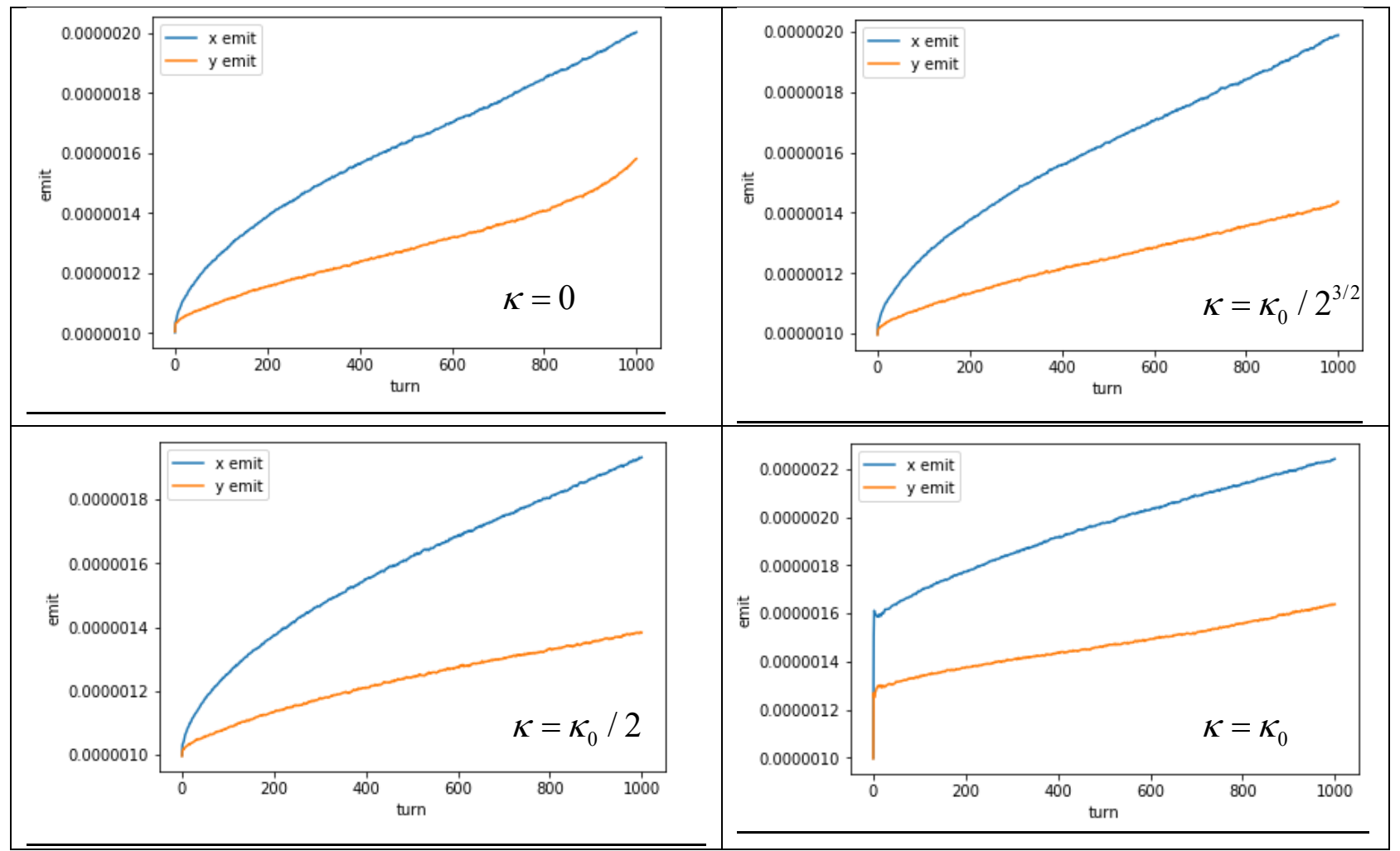

Figure 4. Emittance growth depending on the initial matching.

\section{$\underline{\text { Synergia simulations }}$}

We studied the emittance growth obtained by particle tracking with Synergia for 1000 turns depending on the initial particle distribution obtained from self-consistent $\Sigma$-matrix for various values of parameter $\kappa$. Plots of the r.m.s. emittance growth are presented in Fig. 4. For values $\kappa / \kappa_{0}=1 / 2^{3 / 2}$ and $1 / 2$ there is some reduction in 1000 turns emittance, especially in the vertical 
one, compared to unmatched case $\kappa / \kappa_{0}=0$. With the $\kappa / \kappa_{0}=1$ there was strong initial blowup indicating that matching should be performed for the beam on average, not only for small amplitude particles.

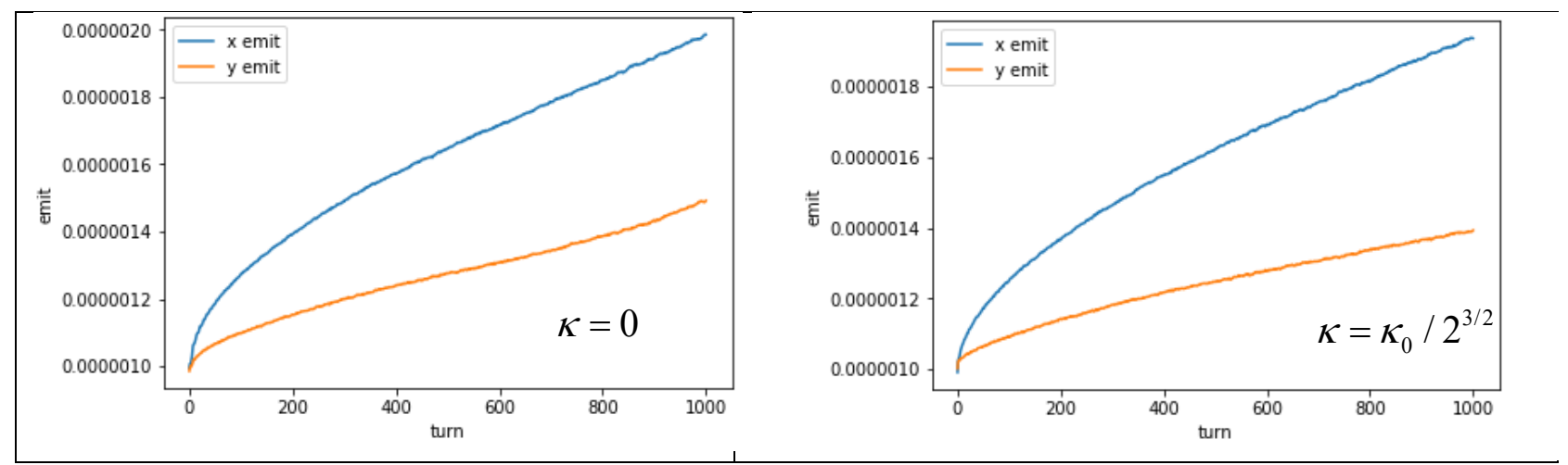

Figure 5. Emittance growth with $4 \sigma$ cut depending on the initial matching.

The effect of matching was actually not as strong as Fig. 4 suggests. If the distributions are cut off at $4 \sigma$ amplitudes, the difference becomes smaller as Fig. 5 testifies where cut emittances are plotted for cases $\kappa / \kappa_{0}=0$ and $1 / 2^{3 / 2}$.

We see that there is no big effect of the initial matching, the case $\kappa / \kappa_{0}=1 / 2$ appears to be close to the optimum. This means that matching for particles with smaller amplitudes is more important than that for the average amplitudes.

\section{References}

[1] Synergia, https://synergia.fnal.gov, see also IPAC2018-THPAF075, Vancouver, CA (2018).

[2] C. Iselin, MAD Physics Guide, http://mad8.web.cern.ch/mad8/

[3] F. Sacherer, PAC71, p.1105 (1971).

[4] Y. Alexahin, F. Schmidt, CERN-ACC-2018-0036, FNAL TM-2688 (2018)

[5] M. D’yachkov, R. Baartman and F.W. Jones, PAC01, p.267 (2001)

[6] R. L. Gluckstern, Phys. Rev. Lett. 73, 1247 (1994). 